\title{
Quantum Capacitance as a Reagentless Molecular Sensing Element
}

\author{
Paulo R. Bueno ${ }^{a, *}$, Flávio C. Bedatty Fernandes ${ }^{a}$ and Jason J. Davis ${ }^{b}$ \\ anstitute of Chemistry, São Paulo State University (UNESP), Araraquara, São Paulo, Brazil. \\ ${ }^{b}$ Department of Chemistry, University of Oxford, South Parks Road, Oxford OX1 3QZ, UK.
}

*prbueno@iq.unesp.br, tel: +55 163301 9642, fax: +55 163322 2308, Instituto de Química, Universidade Estadual Paulista, CP 355, 14800-900 Araraquara, São Paulo, Brazil

\section{Keywords}

Quantum energy transduction, quantum capacitor, molecular layers, molecular assays, graphene, sensor devices, molecular diagnostics.

\section{ABSTRACT (116/150)}

The application of nanoscale capacitance as a transduction of molecular recognition relevant to molecular diagnostics is demonstrated. The energy-related signal relates directly to the electron occupation of quantized states present in readily fabricated molecular junctions such as those presented by redox switchable selfassembled molecular monolayers, reduced graphene oxide or redox-active graphene composite films, assembled on standard metallic or micro-fabricated electrodes. Sensor design is thus based on the response of a confined and resolved electronic density of states to target binding and the associated change in interfacial chemical potential. Demonstrated herein with a number of clinically important markers, this represents a new potent and ultrasensitive molecular detection enabling energy transducer principle capable of quantifying, in a single step and reagentless manner, markers within biological fluid. 


\section{INTRODUCTION}

Despite the limitations imposed by the Heisenberg uncertainty principle, ${ }^{1}$ the occupation of energetically quantized states can be analysed rigorously ${ }^{2}$ and in a manner potentially supporting greater analytical precision than that offered by classical systems. ${ }^{3}$ Derived sensors to date have been physically simple and primarily optical, magnetic in character, ${ }^{4,5}$ or, perhaps most notably, based on field effect phenomena within transistor (FET) architectures. ${ }^{6-11} \mathrm{~A}$ range of nanometer-scale configurations have been reported which are demonstrably capable of supporting analytically rigorous sensing. ${ }^{12-22}$ Within this context graphene has, of course, attracted a lot of recent attention, largely because of its high two-dimensional carrier mobility and available surface area. ${ }^{23-26} \mathrm{~A}$ number of chemically responsive derived FETs have been reported, ${ }^{9,27-29}$ as has its quantum capacitance. ${ }^{30}$ We have ourselves shown that the orbital states presented by electrode-confined redox-active monolayers or two-dimensional graphene films have a cleanly resolvable quantum capacitance. ${ }^{31-34}$ Herein we build upon these principles ${ }^{35,36}$ and observations in showing that this is quantitatively associated with changes in a nanometre-scale density of states (DOS). We thereafter show that this DOS responds predictably and reproducibly to adjacent molecular recognition events in a manner that is applicable to label-free molecular detection (Figure 1) across a range of chemical configurations in an experimentally highly convenient and scaleable format.

We initially remind ourselves of the basis of interfacial capacitance $\left(C_{i}\right)$ in electrochemical environment; a molecular junction immersed into an electrolyte generates a capacitance that is largely modelled by classical means using double layer interfacial capacitance models. The simplest model that works adequately at high $(>0.1 \mathrm{M}$ ) ionic strength is that presented by Gouy-Chapman where the double layer capacitance (per unit of area) is given by $C_{i}=\varepsilon_{\mathrm{r}} \varepsilon_{0} \kappa$, wherein $\varepsilon_{\mathrm{r}}$ is the relative static permittivity (generally referred to as the dielectric constant) of the material, $\varepsilon_{0}\left(\sim 8.85 \times 10^{-12} \mathrm{~F} \mathrm{~m}^{-1}\right)$ is the dielectric constant or dielectric permittivity of vacuum and $\kappa$ is the inverse of Debye length $\left(L_{\mathrm{D}}=1 / \kappa\right)$, classically given by $\kappa=\left[\left(2 e^{2} N\right) /\left(\varepsilon_{0} \varepsilon_{\mathrm{r}} k_{\mathrm{B}} T\right)\right]^{1 / 2} . N$ denotes the density (molar concentration) of ionic charge (positive or negative) in the bulk of the electrolyte, $k_{\mathrm{B}}$ is the Boltzmann constant, and $T$ absolute temperature. Nonetheless this, and any other presented double layer model, fails when electronic states of an adsorbate mix with electrode states. ${ }^{36}$ This is the case, for instance, with electro-active molecular films wherein the innate presence of quantized and redox accessible electronic sites generates additional capacitive effects (usually engrained within what is described as a "pseudo capacitance"). ${ }^{37}$ This additional capacitive effect is associated with the charging of localized chemical states in a way somewhat detached from pure electrostatics. Under such circumstances, then, both ionic and electronic contributions must be considered in a combined electrochemical capacitance ${ }^{37} C_{\bar{\mu}}$ which includes a (DOS based) consideration of Thomas-Fermi screening ${ }^{38}\left[\left(1 / C_{\bar{\mu}}=1 / C_{e}+1 / C_{q}\right)\right.$, where electrostatic $\left(C_{e}\right)$ and quantic $\left(C_{q}\right)$ terms contribute $\left.{ }^{33,39}\right]$ and $\kappa=\left(C_{\bar{\mu}} / \varepsilon_{\mathrm{r}} \varepsilon_{0}\right)^{1 / 2}$.

In reality, then, both ionic (distinguishable particles) and electronic (indistinguishable particles) are engrained in experimentally resolved $C_{\bar{\mu}}$. In the former case, a Boltzmann approximation can be assumed for the occupancy such that $N \propto \exp \left[\bar{\mu} / k_{B} T\right]$. For indistinguishable (electronic) particles $N \propto(1+\exp [\bar{\mu} /$ $\left.\left.k_{B} T\right]\right)^{-1}$. If the former dominates then $\kappa=\left[\left(2 e^{2} N\right) /\left(\varepsilon_{0} \varepsilon_{\mathrm{r}} k_{\mathrm{B}} T\right)\right]^{1 / 2}$ is recovered ${ }^{40}$ from $\kappa=\left(C_{\bar{\mu}} / \varepsilon_{\mathrm{r}} \varepsilon_{0}\right)^{1 / 2}$. Accordingly, the classic double layer phenomenology is thus only a particular form of $C_{\bar{\mu}}$ arising specifically when charging at the interface is exclusively ionic in origin. In situations where there is a significant accessible nanoscale electronic DOS, this dominates in measured $C_{\bar{\mu}}$ and the electrical field screening is primarily governed by electrons. ${ }^{40}$

Under such circumstances, we can directly relate the $1 / C_{\bar{\mu}}$ term, and its relationship to electronic occupation of states, to energy storage ( $E=q V$ and where $q=N e$ is the charge; $e$ is the elementary electron charge) as expressed by ${ }^{35,36}$ 


$$
E=\frac{q^{2}}{2 C_{\bar{\mu}}}=\sum \varepsilon_{i}+\frac{q^{2}}{2 C_{e}}
$$

wherein $\sum \varepsilon_{i}$ is the sum of individual occupied state energies, $\varepsilon_{i}$. If the occupation of states is environmentally responsive then the associated experimentally resolved $C_{\bar{\mu}}$, which directly reports on DOS occupation, responds likewise. ${ }^{33,35}$

We can experimentally measure $C_{\bar{\mu}}$ (in a convenient single contact standard electrochemical junction where electronic DOS dominates) and relate directly to quantised occupancy according to $N \propto 1 / e^{2} \int C_{\bar{\mu}} d V$, wherein $\Delta V=\int_{V_{i}}^{V_{f}} d V$ accounts for potential window being scanned. ${ }^{35} C_{\bar{\mu}}$ is recorded at a fixed frequency low enough for there to be no kinetic limitations (as exemplified further in Figure 3).

This manuscript demonstrates thus that the quantized energy $E \propto 1 / C_{\bar{\mu}}$ of a mesoscopic interface can be directly utilised as a transducer of molecular recognition (with a high sensitivity) with both self-assembled molecular monolayers and reduced graphene oxide composites at both traditional and micro-fabricated electrodes.

(a)

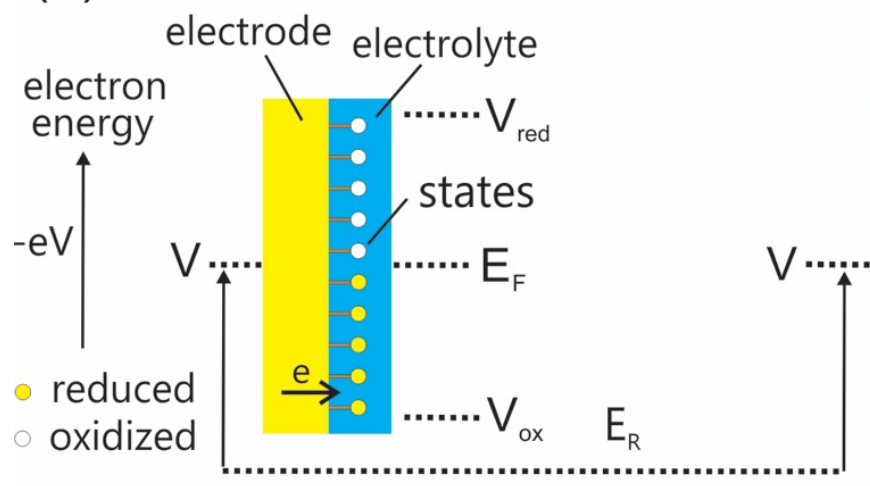

(c)

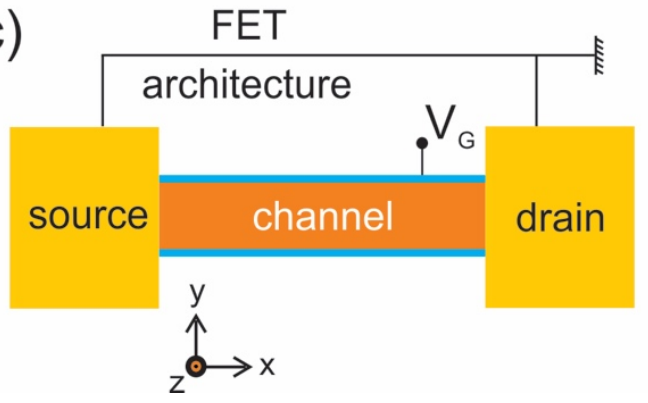

(b)

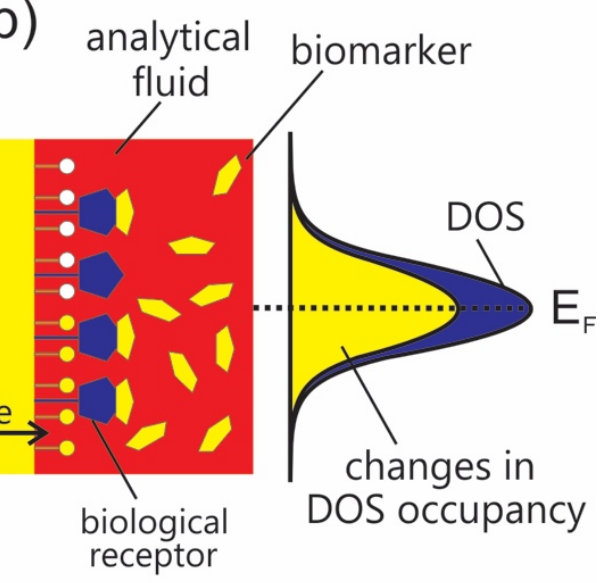

(d)

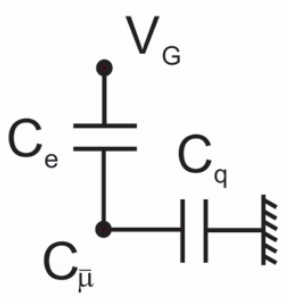

Figure 1. (a) Schematic illustration of the energy levels for an electrochemical junction composed of an electrode (yellow) and a generic electroactive system immersed in electrolyte (blue). The redox reaction free energy is defined as $\Delta G_{r}=e\left(V-V_{r}\right)=E-E_{F}$, where $e$ is the electron charge, $V$ is the electrode over potential. $V_{\text {red }}-V_{o x}$ corresponds to the difference between fully reduced and oxidised states of the junction whereas $E_{R}$ is the reference energy level. (b) Is equivalent to (a) with integrated biological receptors within a mixed junction also containing electro-active states. When a target biomarker is recruited the occupation of the electronic states changes and the experimentally measured change in interfacial energy transduces this through its effect on the film localized DOS. (c) denotes the equivalent operation of a FET functioning under equilibrium conditions, i.e. wherein the electrochemical potentials of the left- and right-hand electrodes are equal. In this situation the electron density in the channel changes as the gate voltage changes. (d) Demonstrates the FET associated equivalent circuit that governs behaviour when only gate voltage $\left(V_{G}\right)$ is varied under equilibrium conditions, that is when the source and drain are poised at the same potential. In such a configuration, the equivalent capacitance is the electrochemical capacitance, measured as $C_{\bar{\mu}}=C_{e} C_{q} /\left(C_{e}+C_{q}\right)$, as discussed in the text.

\section{EXPERIMENTAL METHODS}




\section{Biomarkers and Proteins}

We report herein on the potential application of quantum capacitance (and its associated energy) as a generic transducer at suitably designed interfaces for molecular detection assays. Receptors are coupled to these capacitive interfaces to enable the detection of relevant binding partners such as C-reactive protein (CRP), alpha-synuclein ( $\alpha$-sync) and human prostatic acid phosphatase (PAP). Unless otherwise stated, all proteins used were purchased from Sigma-Aldrich (CRP, anti-CRP, PAP and anti-PAP, BSA and fetuin). In the case of monoclonal anti-prostatic acid phosphatase (PAP, Sigma-Aldrich - product number P5687) specificity was tested by immunoblotting, using denatured-reduced human seminal plasma, wherein the antibody labels the $48 \mathrm{kD}$ subunits of the PAP molecule, but not the prostatic specific antigen (PSA). CRP and goat anti-human CRP were obtained from Sigma Aldrich (product numbers C4063 and C8284), where specificity was confirmed by immune-electrophoresis (IEP) versus human CRP positive serum and purified human CRP. Anti-human alpha-synuclein (Ab-syn) and recombinant alpha-synuclein were purchased from Santa Cruz Biotechnology; the latter was expressed in Escherichia coli and purified according to the supplier. ${ }^{41}$

\section{Preparation of gold interfaces}

All measurements were made at a controlled room temperature of $298 \mathrm{~K}$ and all solutions deoxygenated via bubbling argon and surface purging for the duration of the experiments. Aqueous solutions were prepared using ultrapure water with resistivity not less than $18.2 \mathrm{M} \Omega \mathrm{cm}$ at $298 \mathrm{~K}$ (Millipore Simplicity). All the reagents were purchased from Sigma-Aldrich.

Gold interfaces were initially mechanically polished with aluminum oxide pads through decreasing particle size $(1,0.3$ and $0.05 \mu \mathrm{m})$. Sonication in water was performed between polishing to remove adhered particles. Electrode surfaces were then electrochemically polished in deaerated $\mathrm{NaOH} 0.5 \mathrm{~mol} \mathrm{~L}^{-1}$ (from -0.7 to $-1.7 \mathrm{~V}$ versus $\mathrm{Ag} \mid \mathrm{AgCl}$ at a scan rate of $50 \mathrm{mV} \mathrm{s}^{-1}$ ) and subsequently in deaerated $0.5 \mathrm{~mol} \mathrm{~L}^{-1} \mathrm{H}_{2} \mathrm{SO}_{4}$ (from -0.2 to +1.5 $\checkmark$ versus $\mathrm{Ag} \mid \mathrm{AgCl}$ at a scan rate of $100 \mathrm{mV} \mathrm{s}^{-1}$ ) until the gold reduction peak in cyclic voltammetry stabilized (ca. 50 cycles). Electro-active areas were evaluated by integration of the cathodic peak from the gold electropolishing voltammograms and converted to the real surface area using a conversion factor of $410 \mu \mathrm{C}$ $\mathrm{cm}^{-2}{ }^{42}$ The roughness factor was 1.1 to $1.3\left(0.035\right.$ to $\left.0.041 \mathrm{~cm}^{2}\right)$ throughout.

\section{Procedure for target analyte detection}

In the detection of CRP and alpha Synuclein antibody (Ab-Syn) (see Figure 5) the variation of energy $\Delta E$ upon binding of the electrode poised at the Fermi energy, $E_{F}, 0.45 \mathrm{~V}$ as determined by $\mathrm{CV}$, was used. The receptive species (alpha-synuclein and anti-CRP) were immobilized on 16MHDA/11FcC SAMs by previously discussed standard carbodiimide methods. The interfaces were then immersed in $1 \mathrm{M}$ ethanolamine $(\mathrm{pH}$ of 8.5) to deactivate any unreacted activated carboxylic groups and washed with PBS. Analytical curves were obtained for Ab-syn from $10^{2}$ to $10^{4} \mathrm{pM}$ and 10 to $10^{5} \mathrm{pM}$ for CRP. Impedance-derived capacitance measurements were conducted in a frequency range of from $1 \mathrm{MHz}$ to $10 \mathrm{mHz}$ at the formal potential in triplicate after 30 minutes of incubation in each target concentrations (where the electrodes were washed with PBS, dried and characterized) from where the variance in the energy upon biding was obtained according to Eqn. (1) of the main text. BSA was used in order to evaluate the specificity associated with each functionalized surface. Across the concentration range tested this was accompanied by negligible non-specific binding (see Table 1 of the main text). The detection of CRP and human prostatic acid phosphatase (PAP) were equivalently assessed using mixed monolayers comprising thiolated PEG [poly(ethylene glycol) purchased from Prochimia surfaces, Poland] (for covalent immobilization of the receptive species) and 11-ferrocenyl- 
undecanethiol (1:100)., PEG carboxy terminations activated with EDC/NHS and subsequently immersed in 5 $\mu \mathrm{M}$ solution of anti-CRP or anti-PAP for $3 \mathrm{~h}$.

\section{Impedance-derived nanoscale capacitance measurements}

Cyclic voltammetry and impedance-derived capacitance spectroscopy were carried out using an AUTOLAB (METROHM) potentiostat model PGSTAT30 controlled by NOVA software. A three electrode setup was used for all work, comprising modified gold working electrodes, a platinum mesh counter electrode, and an $\mathrm{Ag} \mid \mathrm{AgCl}, 3 \mathrm{~mol} \mathrm{~L}^{-1} \mathrm{KCl}$ reference. Impedance-derived capacitance spectroscopy was carried out between the frequencies of $1 \mathrm{MHz}$ and $10 \mathrm{mHz}$ with RMS amplitude perturbation of $7 \mathrm{mV} . Z^{\prime}$ and $Z^{\prime \prime}$ values were converted to $C^{\prime \prime}$ and $C^{\prime}$ using the relationships $C^{\prime}(\omega)=Z^{\prime \prime} / \omega|\mathrm{Z}|^{2}$ and $C^{\prime \prime}(\omega)=Z^{\prime} / \omega|\mathrm{Z}|^{2}$. All experiments were carried out at three different electrodes and standard deviation error values calculated from these repeats. All impedance spectroscopic experiments were also checked to satisfy the Kramers-Kronig relationship of timeinvariance. All the DOS curves observed in Figure 2 were constructed by taking potential scans between +0.2 and $+0.8 \mathrm{~V}$ versus $\mathrm{Ag} \mid \mathrm{AgCl}$ with a potential step of $15 \mathrm{mV}$ at a fixed frequency of $80 \mathrm{mHz}$ in supporting electrolyte of $20 \mathrm{mmol} \mathrm{L}^{-1}$ perchlorate tetrabutylammonium (TBA-ClO 4 ) dissolved in acetonitrile and water $(20: 80, v / v)$. Unless otherwise stated, this was the electrolyte used in all DOS measurements, with measurements conducted after the incubation of the receptive interface in PBS containing specific target marker concentrations. The presence of acetonitrile slightly improved redox stability and enables dissolution of $\mathrm{TBA}_{-} \mathrm{ClO}_{4}$. The potential impact of this was evaluated (by circular dichroism spectroscopy - not shown) and confirmed to have no deleterious influence on specific recognition. ${ }^{32,43,44}$ In addition, the resolved equilibrium binding constant $\left(K_{a}\right)$ following this protocol (see Figure ESI-2 and details below) was, within error, the same as that observed in PBS only.

Additional details (such as calibration procedure and determination of the formal potential) of the addressable interfaces utilized herein based on redox and/or receptor modified planar gold electrodes are described in ES-1.

\section{Determination of binding affinity constant}

The Langmuir isotherm model ${ }^{32}$ was utilized in calculating binding affinity constants $\left(K_{a}\right)$. The linearized isotherm was obtained using the ratio between [target] concentration and the transducer signal. The linear form of Langmuir isotherm can be assigned as [target] $/ \Delta S=$ [target] $/ \Delta S_{m}+1 / K_{a} \Delta S_{m}$, where $\Delta S$ and $\Delta S_{m}$ are the signal variation and maximum signal variation, respectively. Here variations of the interfacial energy, $\Delta E$, were used as the transducer signal. The linearized Langmuir isotherm can be constructed by plotting [CRP]/ $\Delta E$ versus [CRP] as shown in Figure ESI-2 (plotted there using data shown in Figure $4 b$ ). $K_{a}$ value of 3.2 $\pm 4 \times 10^{8} \mathrm{~mol}^{-1} \mathrm{~L}$ was obtained as the quotient between the angular $\left(1 / \Delta E_{m}\right)$ and linear $\left(1 / K_{a} \Delta E_{m}\right)$ coefficients.

\section{RESULTS AND DISCUSSION}

Figure $2 a$ shows the variation of DOS shape (as a mean of measurements made across three different electrodes) of 11-(ferrocenyl)undecanethiol, 16-mercaptohexadecanoic acid modified gold electrodes which served as DOS sensors as observed under two different solvent conditions (each electrode was measured in both solvents after equilibration): (i) water (aqueous electrolyte containing $500 \mathrm{mM} \mathrm{NaClO}{ }_{4}$ ) and (ii) dichloromethane (non-aqueous electrolyte containing $100 \mathrm{mM}$ TBA PF 6 ). Over the timescale of analysis, electrodes could be moved between solvent and reanalyzed with no change in the total redox accessible DOS $\left(\sim 1.4 \times 10^{14}\right.$ electrochemical states per $\left.\mathrm{cm}^{-2}\right)$. Note that the Fermi energy of the interfaces fluctuates to sustain constant redox occupancy as local dielectric constant changes. It is worth noting that the influence of local 
dielectric constant is quite different to that associated with a neighbouring molecular recognition (Figure $2 b$ $d$ ); in the latter case the Fermi energy is constant (the dotted line in Figure $2 b$ ) whilst the DOS occupation changes.
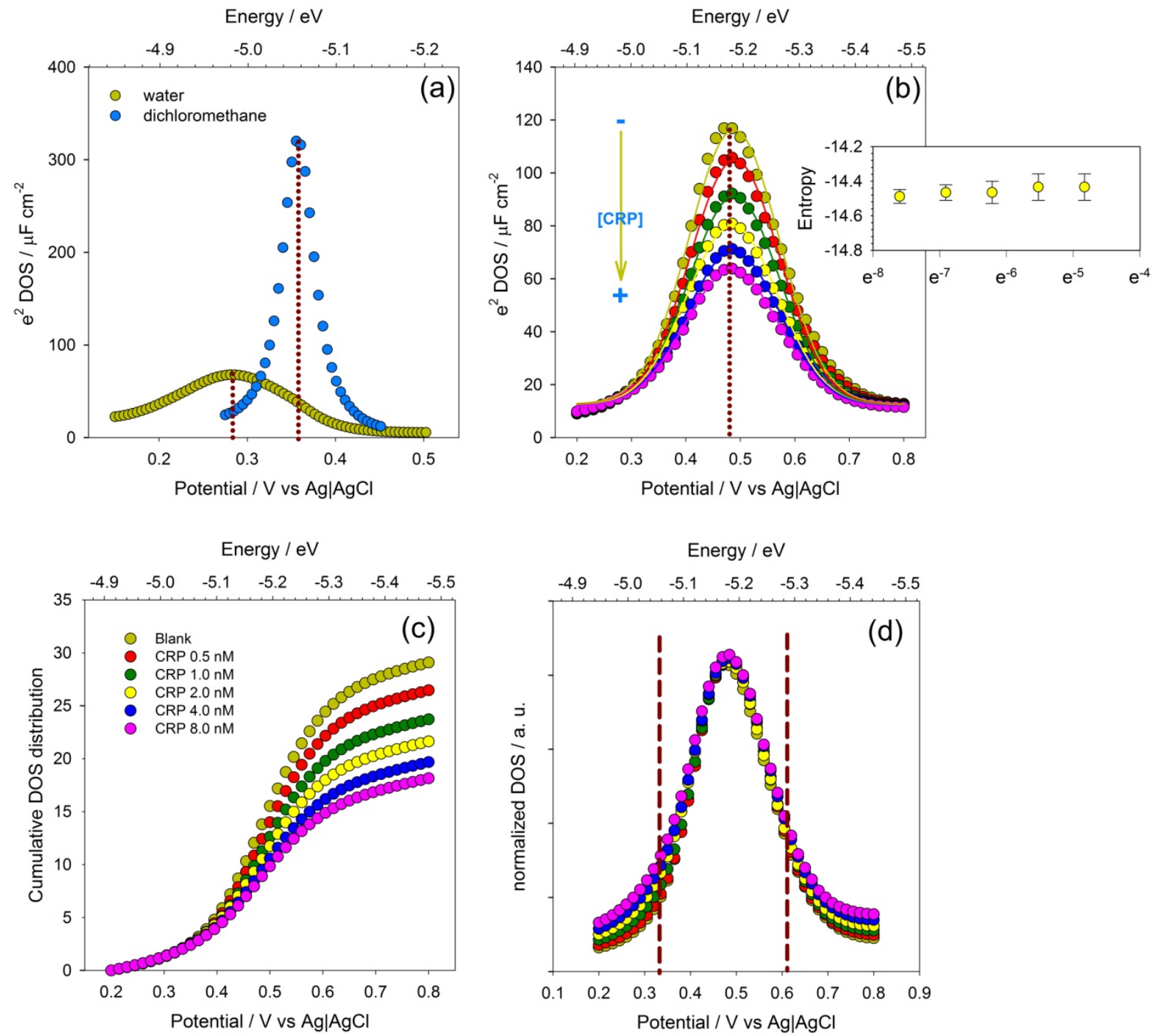

Figure 2. (a) The effect of environment dielectric on resolved DOS distribution of an electroactive molecular layer ${ }^{33}$; solvent polarity tunes both the DOS energetic dispersion and the associated Fermi energy (dotted lines). Notably, the integrated accessible electron density is not affected (being here a constant $\sim 10^{19}$ states per $\mathrm{cm}^{-3}$ ). (b) The responsiveness of redox DOS to molecular recognition within a mixed redox switchable and antibody constrained film (see Figure $1 b$ ). The inlay in (b) shows the invariance in both Fermi energy of the junction ( $0.49 \mathrm{~V}$ versus $\mathrm{Ag} \mid \mathrm{AgCl}$ chemical reference) and state dispersion with $\mathrm{C}$ Reactive Protein target concentration. (c) Cumulative distribution function $\left[\Delta N(V) \propto 1 / e^{2} \int C_{\bar{\mu}} d V\right]$ of electronic states as a function of electrode potential, where the DOS occupancy changes are directly reported. As discussed in the main text $N$ varies as a function of target protein concentration such as $\Delta N$ [target] $\propto \Delta(d N / d E)$ [target]. (d) The density of state function data of $2 b$ normalized for the total state occupancy at each target concentration ( $\Delta N_{t}$ [target]; obtained by integration of the DOS across all relevant energies). The superposition of resulting plots confirms that the energetic change associated with occupancy change, $\Delta E / \Delta N_{t}$ [target] $=\left(e^{2} / C_{\bar{\mu}}\right)$, is constant. All values/curves obtained represent means across triplicate measurements.

The DOS curves were constructed from the electrochemical ${ }^{35,36}$ capacitance following Eqn. (1) as exemplified in Figure $3 a$ (capacitive Nyquist diagram) and $3 b$ (capacitive Bode diagram). In short, this nanoscale capacitance, is measured by impedance-derived capacitance spectroscopy ${ }^{31,36}$ from which the DOS curves (as shown in Figure 2) are obtained at a fixed frequency. This provides the direct-current equivalent 
value of the quantum capacitance obtained from $C^{\prime}$ at this particular fixed frequency $(80 \mathrm{mHz})$. Analysis was confined to this low frequency because $C_{\bar{\mu}}$, as defined previously in Eqn. (1), has a kinetic dependence that can be defined s $^{37}$

$$
C_{\bar{\mu}}(\omega)=\frac{C_{\bar{\mu}}(0)}{1+j \omega \tau}
$$

wherein $\tau$ is the relaxation time of the charging process and $C_{\bar{\mu}}(0)$ is the capacitance at $\omega \rightarrow 0$ in which the $C_{\bar{\mu}}(\omega)=C_{\bar{\mu}}(0)$ such that any measured capacitance is not kinetically limited (equivalent to the DC capacitance, at zero frequency response, that would be obtained). Unless otherwise stated all the capacitive $\left(C_{\bar{\mu}}\right)$ and associated energies $(E)$ measured in the present work were obtained at this frequency (exemplified in Figure 3).
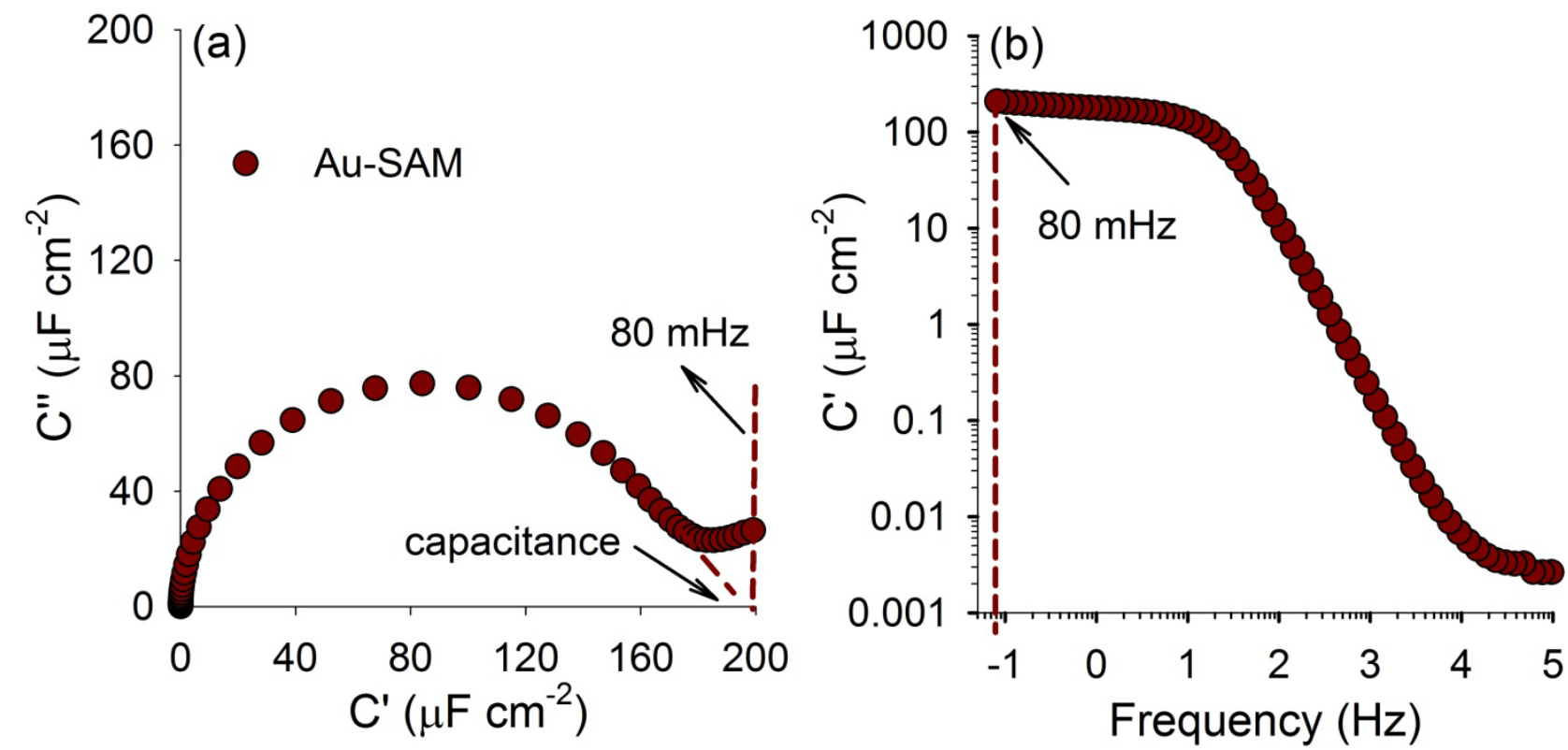

Figure 3. Impedance-derived capacitance measurements made at the Fermi level of the junctions (corresponding to a potential of 0.49 $\checkmark$ versus $\mathrm{Ag} \mid \mathrm{AgCl}$ ), which coincides with the maximum of the DOS curve observed in Figure. (a) The capacitive Nyquist and (b) Bode diagrams show that the DC capacitance is about $200 \mu \mathrm{F} \mathrm{cm}{ }^{-2}$. These analyses are useful in determining the DC electrochemical capacitive [as discussed in the context of Eqn. (2)]. This capacitance can be measured as a function of potential scanned at fixed predetermined frequency of $80 \mathrm{mHz}$ and DOS curves can be constructed as demonstrated in Figure 2. Here the value of $200 \mu \mathrm{F} \mathrm{cm}^{-2}$ correspond to the maximum value of the DOS corresponding to capacitance values measured at the Fermi energy of the junction.

In explaining the origin of DOS analysis, we can now return to Figure $2 a$, showing how change in local dielectric constant translates into a resolved DOS energetic redistribution without a change in total state occupancy $\left[\Delta N_{t}=\left(1 / e^{2}\right) \int_{-\infty}^{+\infty} C_{\bar{\mu}}(V) d V\right.$, as expressed ${ }^{3}$ by the total area of the normal DOS distribution function]. A molecular binding event, however (such as that occurring at a neighbouring receptive site) triggers a resolved change in DOS occupation (Figure $2 b$ ). We can seek to distinguish between these two environmental triggers by looking at the DOS shape (that follows a normal distribution) and its associated energetic spread as expressed through $S[d N / d E]=\ln \sigma_{g} \sqrt{2 \pi e_{n}}$ (the entropy function of the normal DOS distribution), where $e_{n}$ is the base of natural logarithms and $\sigma_{g}$ the standard deviation. In the case of dielectric change, there is a clearly resolved change in dispersion and Fermi energy (Figure 2a). The effects of a local binding event are 
different in that there is an associated change in chemical potential resolvable through DOS occupation or measured $C_{\bar{\mu}}$ ) without change in electronic dispersion (inset Figure $2 b$ ) or in Fermi energy.

From Eqn. (1) the variation in energy per number of particles (the energy/chemical potential change associated with DOS occupancy change) is $d E / d N=N\left(e^{2} / C_{\bar{\mu}}\right)$, from which the associated change in energy and capacitance are quantified as $\Delta E=\left(e^{2} / C_{\bar{\mu}}\right)$ and $C_{\bar{\mu}} / N=e^{2}(d N / d E)$, respectively. If one normalizes the experimentally measured electrochemical capacitance change (which is proportional to the DOS) (across target binding concentrations) for the total number of states $\Delta N_{t}$ [target], that is $\Delta C_{\bar{\mu}} / \Delta N_{t}[$ target] $=$ $\left[e^{2}(d N / d E)\right]$ the responses collapse to constant $e^{2}(d N / d E)$ value (Figure $2 d$ ). Ultimately this confirms that a neighbouring molecular recognition and associated chemical potential change perturbs only occupancy of the DOS (without a change in the entropy associated with electronic states occupancy). This is especially notable close to the electrode Fermi level, wherein the relationship is linear (as expected for an idealised twodimensional electron gas, ${ }^{36,45}$ where $e^{2} / C_{\bar{\mu}}$ is constant). In summary, the electronic DOS, presented by a redox molecular film, responds to a neighbouring molecular recognition such that the resolved electronic charge, $\Delta N$, is proportional to the target/analytic concentration (Figure 4; here the target being CRP).

This DOS based sensing is, of course, a general principle that can be readily extended to the selective detection of other markers alternatively using specific interfacial chemistries (see Figure 5 ). In all cases $\Delta N$ and/or $\Delta E$ remains a linear function of the logarithm of the target concentration (as noted previously that $\Delta E$ [target $] \propto e^{2} / C_{\bar{\mu}}$ [target]; variations rationalized in terms of the effective variation on the chemical energy state of the surface). ${ }^{35}$ Note that occupancy $(\Delta N)$ or chemical potential/energy change $(\Delta E)$ is most sensitive to target binding at potentials close to the Fermi level such that analytical curves ( $\Delta E$ versus logarithm of [target]) can be derived by consideration of changes at this specific energy only (Figures $4 b$ and Figure 5 ).

Assay characteristics are summarized in Table 1 and, in general, lie in accordance with those obtained previously using different label-free transduction methodologies. ${ }^{46,} 47$ Response specificity at mixed alkanethiol interfaces was studied by evaluating $10^{4} \mathrm{pM}$ of BSA in PBS. The percentage change in $\Delta E$ was $2.1 \%$ and $5 \%$ of the electrode response to the same concentration of Syn and CRP, respectively. An equivalent analysis at PEGYlated interfaces was tested using streptavidin and fetuin and was respectively $2.5 \%$ and $4.2 \%$ of the electrode response to the specific targets at same concentration.

Table 1. The figure-of-merits for the chemically modified electrodes and capacitive assays.

\begin{tabular}{ccccc}
\hline & Syn(16MHDA) & CRP(16MHDA) & CRP(PEGlate) & PAP(PEGlate) \\
\hline Linear range (pM) & $10^{2}-10^{4}$ & $10^{1}-5 \times 10^{4}$ & $5 \times 10^{1}-10^{5}$ & $10^{1}-10^{4}$ \\
Sensitivity & $(9,75 \pm 0.04) \times 10^{3}$ & $(9.65 \pm 0.3) \times 10^{3}$ & $(1.58 \pm 0.05) \times 10^{3}$ & $(0.52 \pm 0.02) \times 10^{3}$ \\
R-squared & $0.996 \pm 0.001$ & $0.997 \pm 0.002$ & $0.994 \pm 0.003$ & $0.990 \pm 0.001$ \\
LoD & $143 \mathrm{pM}$ & 5.4 & $28 \mathrm{pM}$ & $11 \mathrm{pM}$ \\
$\left(K_{a}\right)$ & $(1.58 \pm 0.08) \times 10^{9}$ & $(1.27 \pm 0.16) \times 10^{9}$ & $(2.6 \pm 0.5) \times 10^{9}$ & $(3.6 \pm 0.2) \times 10^{9}$ \\
${\text { Specificity }(\%)^{*}}^{2.1}$ & 5 & 2.5 & 4.2 \\
\hline
\end{tabular}

${ }^{\#}$ Measured as $\left(\Delta E\left(\mathrm{~F} \mathrm{~cm}^{-2}\right)^{-1} / \mathrm{pM}\right)$. *Percentage relative to the electrode response for the target. 

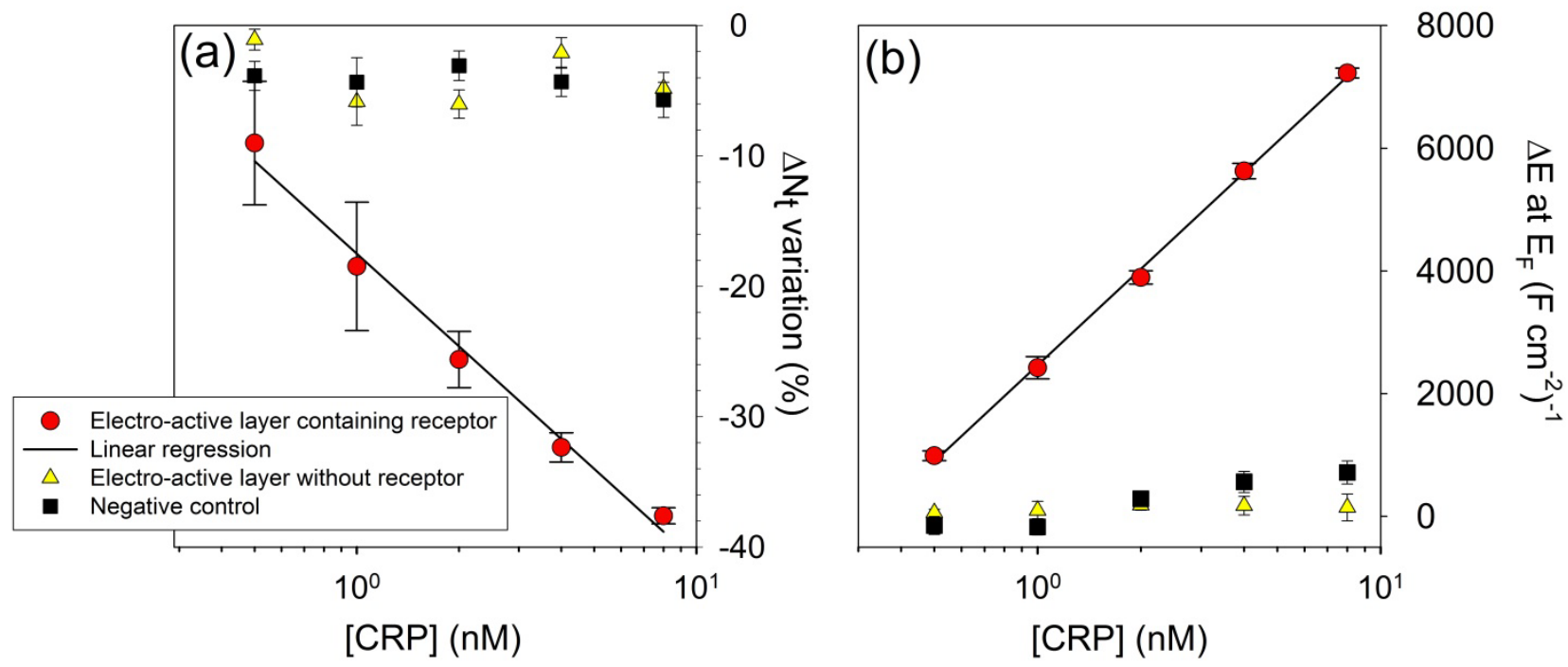

Figure 4. (a) The linear relationship between the variation of total electron density obtained from DOS integration $\left(\Delta N_{t}=\right.$ $\left.\left(1 / e^{2}\right) \int_{-\infty}^{+\infty} C_{\bar{\mu}}(V) d V\right)^{35}$ in the entire interval of potential shown in Figure $2 b$ as a function of target CRP concentration at a redox active SAM-anti-CRP modified gold electrode. The resolved redox DOS is unresponsive both to an equivalent concentration of a negative control (black square) (here Fetuin-A) and to CRP in the absence of a specific antibody receptor (yellow triangles). From the specific response, binding affinities are readily resolved from subsequent fitting to a Langmuir isothermal model (see ESI document for more detail); here $3.2 \pm 4 \times 10^{8} \mathrm{~mol}^{-1} \mathrm{~L}$, comparable to that obtained by other methods for the same interfacial immuno-complexation. ${ }^{48}$ (b) It is equivalently possible to generate analytical curves using the local recognition induced energy change $(\Delta E)$, as resolved at a single potential, $0.49 \mathrm{~V}$ versus $\mathrm{Ag} \mid \mathrm{AgCl}$, the Fermi energy $\left(E_{F}\right)$ of the junction. Energy and occupancy are anti-correlated. The error bars represent standard deviation across three independent receptive junctions.

The general principles of utilizing the resolved sensitivity of a surface confined electrode-chargeable DOS as a transducer signal of captured target can be extrapolated readily to antibody-modified non-redox active graphene derived interfaces assembled on metallic electrodes as shown in Figure 6 . In Figure $6 a$ glassy carbon electrode (GCE) interfaces were utilized. In Figures $6 b$ and $6 c$ the optimized redox-composite (see in Figure ESI-3a) was applied to gold micro-fabricated electrodes. To assure the proper translation of the receptive interface from gold-disk (measurements shown in ESI-3a) to gold-micro-fabricated electrodes the latter was tested in PBS following the previous procedures (and demonstrated in measurements shown in Figure ESI-3b) made in the modified electrodes containing redox-active groups in gold-disk electrodes (as those made previously in Figures 2, 4 and 5). The LoD obtained was 16.8 $\pm 1.2 \mathrm{pM}$ across three different electrodes which compares quite well with those LoD of $21.6 \pm 5.2$ obtained at gold-disk electrodes. The LoD obtained in serum was resolved as $56.4 \pm 7.8 \mathrm{pM}$. To confirm specificity this receptive interface was incubated with human serum albumin (HSA from Sigma-Aldrich) in PBS with HSA at concentrations ranging from $50 \mathrm{pM}$ to $10^{5} \mathrm{pM}$ wherein the relative response in percentage $\Delta E$ oscillated between $-2.2 \pm 1.0 \%$ to $1.6 \pm 1.7 \%$ (inside of the noise of equipment and thus within experimental error).

We have shown, then, that the mesoscopic principles active at a redox-confined surface ${ }^{37}$ extend readily to non-redox active interfaces where an accessible DOS is confined to nanometer scale entities and highly responsive to environment. ${ }^{40}$ This was exemplified here through the use of electrode immobilized and antibody modified reduced graphene oxide films (Figure $6 a$ ). Interfaces generated at gold-micro-fabricated electrodes were applied to quantify the detection of CRP in serum across clinically relevant target concentrations of $5 \times 10^{3}$ to $10^{5} \mathrm{pM}$ (as shown in Figure $6 \mathrm{c}$ ). ${ }^{49,50} \mathrm{In}$ this case, the CRP recruiting interface ( $\mathrm{Au} / 11-\mathrm{FcC} / \mathrm{GO} / \mathrm{CBMA} / \mathrm{Ab}-\mathrm{CRP}$ - see more details about this interface in ESI-3) was initially incubated in native, unspiked, serum. This was carried out in order to generate a baseline response (Figure $6 b$ ) prior to additional spiking with target. 

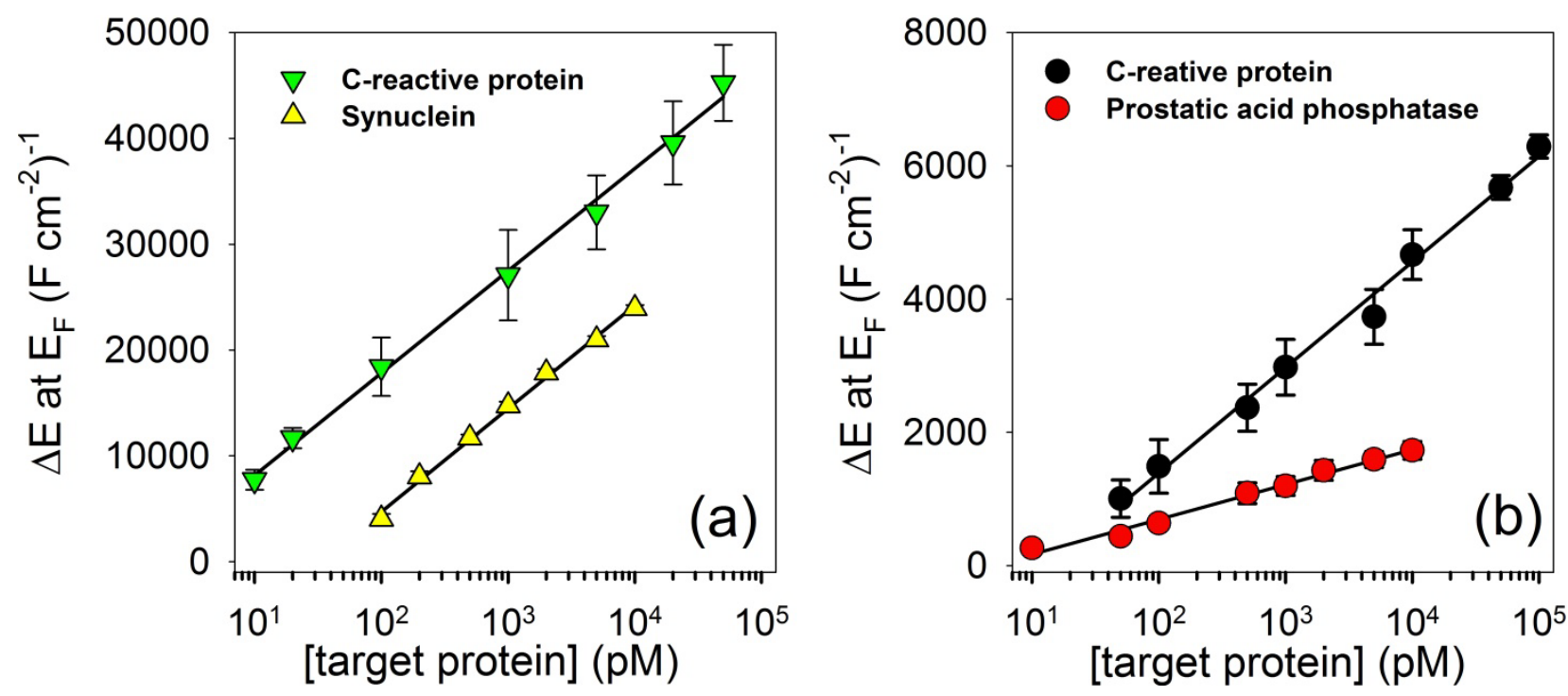

Figure 5. Analytical curves obtained by plotting variation of the energy of the accessible interfacial states $(\Delta E)$ at their fixed Fermi energy $\left(E_{F}\right)$ for a range of clinically relevant markers [here C-reactive protein, prostatic acid phosphatase and $\alpha$-synuclein, respectively markers for cardiac and general inflammation, ${ }^{51}$ prostate cancer ${ }^{52}$ and Parkinson's disease ${ }^{53}$ ]. The transduction principles active here are independent of specific film composition; the receptive films utilized are mixed thiolated ferrocene (providing the accessible responsive DOS) and thiolated alkyl or pegylated carboxylate. Assay sensitivity shows some predictable dependence on film (see ESI for more experimental detail). Error bars represent standard deviations derived from measurements conducted at three independent electrodes.

Finally, we demonstrate that the operational principles associated with these capacitive spectroscopy resolved DOS assays are analogous to those operating in FET-based devices under equilibrium conditions (see Figure $1 c$ and legend), wherein the quantum capacitance behaves in a manner equivalent to the channel states of FET devices. We first observe that the difference between the potential in the channel/bridge $\left(V_{c}\right)$ and in the gate $\left(V_{G}\right)$ is dependent on $N$ as $V_{c}-V_{G}=-\left(e N / C_{e}\right)$ (in electro-active molecular layers this corresponds to the potential difference between the local potential of the accessible states ${ }^{54,55}$ and the external potential in the electrolyte). The gate capacitance is thus $d q / d V_{G}=\left(d q / d V_{c}\right)\left(d V_{c} / d V_{G}\right)$. By noting that $d q / d V_{G}$ is $C_{\bar{\mu}}$ and $d q / d V_{c}$ is the quantum capacitance $\left(C_{q}\right), d q / d V_{G}$ rearranges to give [see ESI-4 for more detail]

$$
\frac{q^{2}}{C_{\bar{\mu}}}=q^{2}\left(\frac{d V_{G}}{d q}\right)=q^{2}\left(\frac{C_{q}+C_{e}}{C_{q} C_{q}}\right)=q^{2}\left(\frac{1}{C_{e}}+\frac{1}{C_{q}}\right)
$$

an expression explicitly equivalent to Eqn. (1). Eqn. (3) implicitly demonstrates that, in experimentally accessing $C_{\bar{\mu}}$, the thermodynamic properties of the junctions are trackable, potentially as a function of a neighbouring/integrated target recognition. This is accessible through $\Delta G=e^{2} / C_{\bar{\mu}}=-k_{B} T \ln K_{a}[R]$ (wherein $[R]$ is the concentration of receptor and $\Delta G$ the free energy of the electrochemical reaction) which additionally implies the observed semi-logarithmic linear relationship (shown in Figures $4 b, 5$ and 6) resolved under equilibrium binding conditions (the Langmuir adsorption model). It is worth noting that these binding induced changes in electronic free energy are accompanied by associated change in state occupation (discharging /charging $C_{\bar{\mu}}$ and de-populating/populating states) such that the Fermi energy does not change (Figure $2 b$ ). In summary, the capacitive DOS sensing introduced here is equivalent to those operating in microfabricated FET devices but, significantly, is both highly chemically tailorable and requires just one contact; Figures 5 and $6 a$ show how the resolved electrochemical energy ${ }^{40}$ (electronically dominated) of an anti-CRP antibody responds to clinically relevant levels of CRP at both macro-disk and micro-fabricated disposable electrode arrays, respectively. Figure $6 a$ specifically reports on a functionalized reduced graphene oxide and 
composite interfaces responds to clinically relevant levels of CRP at a micro-fabricated disposable electrode arrays thus demonstrating the extended technological applicability of the principles reported in the present manuscript. $^{56,57}$
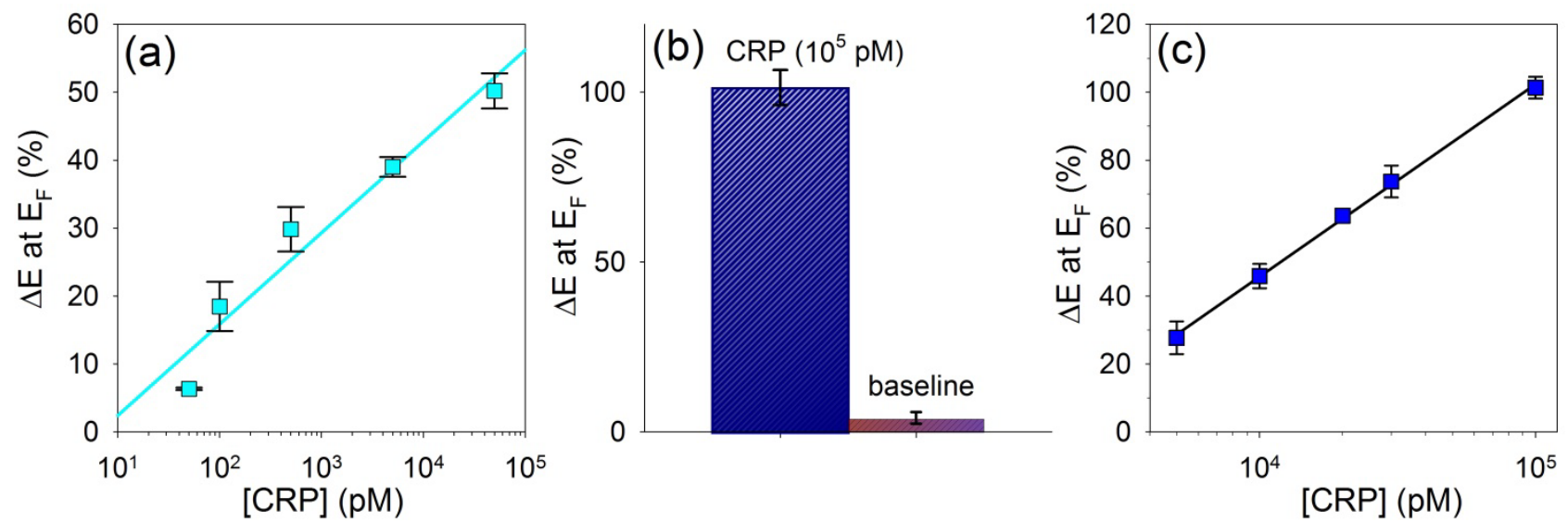

Figure 6. (a) Capacitance resolved variations in $\Delta E \propto \Delta\left(1 / C_{\bar{\mu}}\right)$ for electrochemically reduced graphene oxide multilayers functionalized with anti-CRP antibodies. (b) Demonstrative selectivity obtained at micro-fabricated arrays modified with anti-CRP-modified redoxmodified graphene oxide; here the response to $10^{5} \mathrm{pM}$ CRP spiked into serum is shown comparatively to a that at native serum prior to spiking (see ESI) (c) equivalent to (a) is the analytical curve comprising the linear relationship between $\Delta E$ and the logarithm of CRP concentration in serum as resolved at redox and graphene oxide modified micro-fabricated gold electrode arrays (detection limit 55 $\mathrm{pM}$, linear analytical range is $>10^{3} \mathrm{pM}<10^{5} \mathrm{pM}$, relevant to clinical need. ${ }^{49,57} \mathrm{In}$ (c) the errors bars are standard deviations calculated across three measurements performed at the same disposable array (see ESI document).

\section{CONCLUSION AND FINAL REMARKS}

To conclude, we show herein that a surface confined and electronically addressable DOS (comprising either redox switchable centres or appropriately immobilized mesoscopic units) contributes to a readily resolved quantum capacitance. This charging reports on the occupancy of quantized states and responds sensitively to changes in local chemical potential. By appropriately introducing receptors this entirely reagentless sensing becomes highly specific and very sensitive. This transduction mechanism operates in a manner analogous to FET devices but in a markedly more experimentally accessible and chemically flexible manner. We believe that the combination of this approach with standard microfluidic and micro-fabrication methods offers much to both diagnostics and chemical sensing generally.

\section{Acknowledgements}

The authors gratefully acknowledge Royal Society and FAPESP for financial support.

\section{References}

1. W. Heisenberg, Zeitschrift für Physik, 1927, 43, 172-198.

2. N. Thomas-Peter, B. J. Smith, A. Datta, L. Zhang, U. Dorner and I. A. Walmsley, Phys. Rev. Lett., 2011, $107,113603-113607$.

3. V. Giovannetti, S. Lloyd and L. Maccone, Science (New York, N.Y.), 2004, 306, 1330-1336.

4. C. Aiello, M. Hirose and P. Cappellaro, Nat. Commun., 2013, 4.

5. N. Maccaferri, K. E. Gregorczyk, T. V. A. G. de Oliveira, M. Kataja, S. P. van Dijken, Z.;, A. Dmitriev, J. Åkerman, M. Knez and P. Vavassori, Nature communications, 2014, 6, 6150.

6. M. Muccini, Nature Materials, 2006, 5, 605-613.

7. I. Ferain, C. A. Colinge and J.-P. Colinge, Nature, 2011, 479, 310-316.

8. A. M. lonescu and H. Riel, Nature, 2011, 479, 329-337. 
9. $\quad$ F. Schwierz, Nature nanotechnology, 2010, 5, 487-496.

10. B. Wang, J. C. Cancilla, J. S. Torrecilla and H. Haick, Nano Lett., 2014, 14, 933-938.

11. S. Kubatkin, A. Danilov, M. Hjort, J. Cornil, J.-L. Bredas, N. Stuhr-Hansen, P. Hedegard and T. Bjørnholm, Nature, 2003, 425, 698-701.

12. N. Liu, M. L. Tang, M. Hentschel, H. Giessen and A. P. Alivisatos, Nature Materials, 2011, 10, 631-636.

13. B. L. Allen, P. D. Kichambare and A. Star, Adv. Mat., 2007, 19, 1439-1451.

14. K. Bradley, M. Briman, A. Star and G. Gruner, Nano Lett., 2004, 4, 253-256.

15. R. J. Chen, S. Bangsaruntip, K. A. Drouvalakis, N. W. S. Kam, M. Shim, Y. M. Li, W. Kim, P. J. Utz and H. J. Dai, Proc. Natl. Acad. Sci. U.S.A., 2003, 100, 4984-4989.

16. X. F. Guo, L. M. Huang, S. O'Brien, P. Kim and C. Nuckolls, J. Amer. Chem. Soc., 2005, 127, 15045-15047.

17. A. Merkoci, M. Pumera, X. Llopis, B. Perez, M. del Valle and S. Alegret, Trac-Trends Anal. Chem., 2005, 24, 826-838.

18. M. Shim, A. Javey, N. W. S. Kam and H. J. Dai, J. Amer. Chem. Soc., 2001, 123, 11512-11513.

19. A. Star, V. Joshi, S. Skarupo, D. Thomas and J.-C. P. Gabriel, J. Phys. Chem. B, 2006, 110, 21014-21020.

20. M. Trojanowicz, Trac-Trends Anal. Chem., 2006, 25, 480-489.

21. J. N. Anker, W. P. Hall, O. Lyandres, N. C. Shah, J. Zho and R. P. Van Duyne, Nature Materials, 2008, 7, 442-453.

22. B. Luk'yanchuk, N. Zheludev, S. A. Maier, N. J. Halas, P. Nordlander, H. Giessen and C. T. Chong, Nature Materials, 2010, 9, 707-715.

23. L. Zhang and X. Zhao, Chem. Soc. Rev., 2009, 38, 2520-2531.

24. J. Jung, I. Sohn, D. Kim, B. Kim, M. Fang and N. Lee, Carbon, 2013, 62, 312-321.

25. J. Chang, S. Mao, Y. Zhang, S. Cui, G. Zhou, X. Wu, C. Yang and J. Chen, Nanoscale, 2013, 5, 3620-3626.

26. S. Liu and X. Guo, Npg Asia Mat., 2012, 4, 1-10.

27. A. Javey, J. Guo, Q. Wang, M. Lundstrom and D. Hongjie, Nature, 2003, 424, 654-657.

28. W. Liang, M. P. Shores, M. Bockrath, J. R. Long and H. Park, Nature 417, 2002, 417, 725-729.

29. M. Chhowalla, H. S. Shin, G. Eda, L.-J. Li, K. P. Loh and H. Zhang, Nature Chemistry, 2013, 5, 263-275.

30. J. Xia, F. Chen, J. Li and N. Tao, Nature Nanotech., 2009, 4, 505-509.

31. F. C. B. Fernandes, M. S. Góes, J. J. Davis and P. R. Bueno, Biosens. Bioelectron., 2013, 50, 437-440.

32. F. C. B. Fernandes, A. Santos, D. C.Martins, M. S. Góes and P. R. Bueno, Biosens. Bioelectron., 2014, 57, 96-102.

33. P. R. Bueno and J. J. Davis, Anal. Chem., 2014, 86, 1337-1341.

34. P. R. Bueno, J. J. Davis and G. Mizzon, J. Phys. Chem. C, 2012, 116, 8822-8829.

35. D. A. Miranda and P. R. Bueno, Phys. Chem. Chem. Phys., 2016, 18, 25984-25992.

36. P. R. Bueno, G. T. Feliciano and J. J. Davis, Phys. Chem. Chem. Phys., 2015, 17, 9375-9382.

37. P. R. Bueno, T. A. Benites and J. J. Davis, Scientific Reports, 2016, 6, 18400.

38. H. Haug and S. W. Kock, Quantum theory of the optical and electronic properties of semiconductors, World Scientific Publishing, London, 2004.

39. S. Ilani, L. A. K. Donev, M. Kindermann and P. L. McEuen, Nature Physics, 2006, 2, 687-691.

40. F. A. Gutierrez, F. C. B. Fernandes, G. A. Ribas and P. R. Bueno, Phys. Chem. Chem. Phys., 2017, 67926806.

41. V. N. Uversky, Journal of Biomolecular Structure and Dynamics, 2003, 21, 211-234.

42. S. Trasatti and O. A. Petrii, Pure Appl. Chem., 1991, 63, 711-734.

43. F. C. B. Fernandes, A. V. Patil, P. R. Bueno and J. J. Davis, Analytical Chemistry, 2015, 87, 12137-12144.

44. A. Santos, J. P. Piccoli, N. A. Santos, E. M. Cilli and P. R. Bueno, Biosensors \& Bioelectronics, 2015, 68, 281-287.

45. S. Luryi, Applied Physics Letters, 1988, 52, 501.

46. T. Bryan, L. Xiliang, L. Forsgren, L. A. Morozova-Roche and J. J. Davis, Chemical Science, 2012, 3, 34683473.

47. T. Bryan, X. Luo, P. R. Bueno and J. J. Davis, Biosensors and Bioelectronics, 2013, 38, 94-98.

48. H. Hennessey, N. Afara, S. Omanovic and A. L. Padjen, Anal. Chim. Acta, 2009, 643, 45-53. 
49. N. Sattar, A. Gaw, O. Scherbakova, I. Ford, D. S. J. O’Reilly, S. M. Haffner, C. Isles, P. W. Macfarlane, C. J. Packard, S. M. Cobbe and J. Shepherd, Circulation, 2003, 108, 414-419.

50. P. M. Ridker, J. E. Buring, N. R. Cook and N. Rifai, Circulation, 2003, 107, 391-397.

51. M. P. Pepys, G. M. Hirschfield, G. A. Tennent, J. R. Gallimore, M. C. Kahan, M. Bellotti, P. N. Hawkins, R. M. Myers, M. D. Smith, A. Polara, A. J. A. Cobb, S. V. Ley, J. A. Aquilina, C. V. Robinson, I. Sharif, G. A. Gray, C. A. Sabin, M. C. Jenvey, S. E. Kolstoe, D. Thompson and S. P. Wood, Nature, 2006, 440, 12171221.

52. M. I. Hassan, A. Aijaz and F. Ahmad, Expert Rev. Anticancer Ther., 2010, 10, 1055-1068.

53. F. Soldner, Y. Stelzer, C. S. Shivalila, B. J. Abraham, J. C. Latourelle, M. I. Barrasa, J. Goldmann, R. H. Myers, R. A. Young and R. Jaenisch, Nature, 2016, 533, 95-99.

54. A. D. McNaught and A. Wilkinson, Blackwell Scientific Publications, Oxford, Second Edition edn., 1997. 55. unpublished work.

56. T. A. Pearson, G. A. Mensah, R. W. Alexander, A. J. L.;, R. O. C. Canon, M.;, Y. Y. Fadl, S. P. Fortmann, Y. Hong, G. L. Myers, N. Rifai, S. C. J. Smith, K. Taubert, R. P. Tracy and F. Vinicor, Circulation, 2003, 107, 499-511.

57. P. M. Ridker, J. E. Buring, N. R. Cook and N. Rifai, Circulation, 2003, 107, 391-397.

\section{AUTHOR CONTRIBUTION}

P. R. B. and J. J. D. wrote the manuscript. P. R. B. worked on theoretical analysis. P. R. B. and F. C. B. F. prepared Figures and support information. All authors proofread the manuscript. F. C. B. F. provided raw experimental data. 